\title{
Universal Parametric Correlations of Conductance Peaks in Quantum Dots
}

\author{
Y. Alhassid and H. Attias \\ Center for Theoretical Physics, Sloane Physics Laboratory, \\ Yale University, New Haven, CT 06520, U.S.A.
}

(Submitted 21 February 1995)

\begin{abstract}
We compute the parametric correlation function of the conductance peaks in chaotic and weakly disordered quantum dots in the Coulomb blockade regime and demonstrate its universality upon an appropriate scaling of the parameter. For a symmetric dot we show that this correlation function is affected by breaking time-reversal symmetry but is independent of the details of the channels in the external leads. We derive a new scaling which depends on the eigenfunctions alone and can be extracted directly from the conductance peak heights. Our results are in excellent agreement with model simulations of a disordered quantum dot.
\end{abstract}

PACS numbers: 05.40.+j, 05.45+b, 72.20.My, 73.20.Dx

Typeset using REVTEX 
Quantum dots [1,2] are semiconductor heterostructures that confine about 100 electrons by an electrostatic potential to isolated islands with a typical size of less than a micron. The dots can be weakly coupled via tunnel barriers to external leads in order to study their transport properties. For sufficiently low temperatures the conductance of the dot exhibits equally spaced peaks with increasing gate voltage, where each successive peak corresponds to a tunneling of a single electron into the dot. This occurs when the increase in the Fermi energy in the leads matches the energy required to charge the dot by one additional electron. The suppression of tunneling between the peaks by Coulomb repulsion is known as Coulomb blockade. A striking feature of these resonances is the irregular dependence of their amplitudes on controllable parameters, such as the shape of the dot or an external magnetic field. These fluctuations have recently been accounted for by a statistical theory [3] based on the assumption that the Hamiltonian of the dot can be described by random-matrix theory (RMT) due to irregularities in the confining potential which give rise to a chaotic classical dynamics. RMT description is suitable also for dots with weak impurity disorder since the same conductance distribution is obtained when the dot is modelled by a random potential [4].

Recent experiments have been probing the conductance of quantum dots as an external parameter is varied. In this paper we study the correlation between conductance peak amplitudes which belong to different values of the parameter. Very little is known about this parametric correlator in the regime of isolated resonances, in contrast with the overlapping resonance regime characteristic of open dots where correlations versus energy and magnetic field were investigated both theoretically (for a large number of channels) [5],6] and experimentally [7]. By casting the peak correlator in the framework of the Gaussian random-matrix process (GP) [8], we demonstrate its universality upon an appropriate scaling of the parameter and compute its universal form. Our main results for the conductance correlator are the approximate expression (17) and its exact short-distance behavior (14). We also derive an alternative parameter scaling which is extracted directly from the measured conductance peaks according to Eq. (15). Its universal ratio to the usual level velocity scaling (4) [9] is 
given by Eqs. (11) and (12) for the orthogonal and unitary cases, respectively.

We focus on the temperature regime $\Gamma \ll k T<\Delta$, typical of experiments [1,2], where $\Delta$ is the mean resonance spacing. In this regime only one quasi-bound state (usually the ground state) of the electron gas in the dot contributes to each conductance peak [10,11], whose width is $\sim k T$. The peak amplitude that corresponds to a level $\lambda$ is given by [11]

$$
G_{\lambda}=\frac{e^{2}}{h} \frac{\pi}{2 k T} \frac{\Gamma_{\lambda}^{l} \Gamma_{\lambda}^{r}}{\Gamma_{\lambda}^{l}+\Gamma_{\lambda}^{r}},
$$

where $\Gamma_{\lambda}^{l(r)}$ is the total decay width from level $\lambda$ into the left (right) lead. Each lead can support several open channels so that $\Gamma_{\lambda}^{l(r)}=\sum_{c} \Gamma_{c \lambda}^{l(r)}$, where $\Gamma_{c \lambda}^{l(r)}$ is the partial decay width into channel $c$. A suitable formalism that relates the level width to the dot's eigenfunction is the $R$-matrix theory [12], according to which $\Gamma_{c \lambda}=\left|\gamma_{c \lambda}\right|^{2}$ where

$$
\gamma_{c \lambda}=\left(\hbar^{2} k_{c} P_{c} / m\right)^{1 / 2} \int d S \Phi_{c}^{*} \Psi_{\lambda}
$$

is the partial width amplitude, $k_{c}$ is the channel momentum and $P_{c}$ is a penetration factor to tunnel through the barrier. Here $\Psi_{\lambda}$ is the dot eigenfunction, $\Phi_{c}$ is the transverse wavefunction in the lead corresponding to an open channel $c$, and the integral extends over the cross-section of the lead at its end attached to the dot.

The connection with RMT [3] is made by assuming that the statistical properties of the dot's Hamiltonian are well described by a $N \times N$ random matrix $H$ taken from the appropriate Gaussian ensemble (GE). The dot's eigenfunction $\Psi_{\lambda}$ is related to the $\lambda$-th eigenvector of $H, \psi_{\lambda \mu}$, via the expansion $\Psi_{\lambda}(\vec{r})=\sum_{\mu=1}^{N} \psi_{\lambda \mu} \rho_{\mu}(\vec{r})$, where $\rho_{\mu}(\vec{r})$ are a set of solutions of Schrödinger's equation at energy $E_{\lambda}$ inside the dot. Omitting the subscript $\lambda$, the partial width amplitude (2) can be written as a scalar product $\gamma_{c}=\sum_{\mu} \phi_{c \mu}^{*} \psi_{\mu} \equiv\left\langle\phi_{c} \mid \psi\right\rangle$, where $\phi_{c \mu} \equiv\left(\hbar^{2} k_{c} P_{c} / m\right)^{1 / 2} \int d S \Phi_{c} \rho_{\mu}^{*}$. The correlations between the $\gamma_{c}$ define the channel correlation matrix $M$, and are given by the scalar products of the channel vectors $M_{c c^{\prime}} \equiv$ $\overline{\gamma_{c}^{*} \gamma_{c^{\prime}}}=\frac{1}{N}\left\langle\phi_{c} \mid \phi_{c^{\prime}}\right\rangle$. The channels can have any degree of correlation among them and different decay widths into them, hence $\phi_{c}$ are in general not orthogonal and have different norms. 
In order to discuss the correlation between conductances at different shapes or external fields we use the framework of the GP, which generalizes Dyson's GE to describe statistical properties of systems that depend on a parameter [8, [13]. A GP is a set of random $N \times N$ matrices $H(x)$ whose elements are distributed at each $x$ according to the appropriate GE with a prescribed correlation among elements at different values of $x$ :

$$
\begin{aligned}
\overline{H_{\lambda \sigma}(x)} & =0, \\
\overline{H_{\lambda \sigma}(x) H_{\mu \nu}\left(x^{\prime}\right)} & =\frac{a^{2}}{2 \beta} f\left(x-x^{\prime}\right) g_{\lambda \sigma, \mu \nu}^{(\beta)},
\end{aligned}
$$

where $g_{\lambda \sigma, \mu \nu}^{(\beta=1)}=\delta_{\lambda \mu} \delta_{\sigma \nu}+\delta_{\lambda \nu} \delta_{\sigma \mu}$ for the Gaussian orthogonal process (GOP) and $g_{\lambda \sigma, \mu \nu}^{(\beta=2)}=$ $2 \delta_{\lambda \nu} \delta_{\sigma \mu}$ for the Gaussian unitary process (GUP). Assuming that the leading order of $f$ is $f \approx 1-\kappa\left(x-x^{\prime}\right)^{2}$, we have shown [8] that $\kappa=\beta \frac{\pi^{2}}{4} \frac{1}{N} \overline{\left(\partial E_{\lambda} / \partial x\right)^{2}} / \Delta^{2}$ which suggests scaling $x$ by the RMS of the level velocity as originally discussed in [9]:

$$
\left.x \rightarrow \bar{x}=\left[\overline{\left(\partial E_{\lambda} / \partial x\right.}\right)^{2} / \Delta^{2}\right]^{1 / 2} x \equiv \sqrt{D} x .
$$

After the scaling (4) $f \approx 1-\beta \frac{\pi^{2}}{4}\left(\bar{x}-\bar{x}^{\prime}\right)^{2} / N$ becomes independent of the non-universal quantity $\kappa$. The parametric correlator for any observable, being determined by $N(1-f)$, is then a universal function of $\bar{x}-\bar{x}^{\prime}$ [8]. This applies not only to spectral correlators [9] but also to those involving the eigenfunctions. In particular, the conductance peak correlator

$$
c_{G}\left(x-x^{\prime}\right) \equiv \overline{\tilde{G}(x) \tilde{G}\left(x^{\prime}\right)}, \quad \tilde{G}(x)=\frac{G(x)-\bar{G}}{\left(\overline{G^{2}}-\bar{G}^{2}\right)^{1 / 2}}
$$

becomes universal upon the scaling (4) for all dots characterized by the same channel correlation matrix $M$.

The case of a left-right symmetric dot is particularly simple since $\Gamma^{l}=\Gamma^{r} \equiv \Gamma$ and the conductance peak correlator reduces to the width correlator $\overline{\tilde{\Gamma}(x) \tilde{\Gamma}\left(x^{\prime}\right)}$. We note that the correlation matrix $M_{c c^{\prime}}$ is Hermitean and positive definite, and hence can be transformed into a diagonal form which defines a set of orthonormal eigenchannel vectors $\hat{\phi}_{c}$. Expressing the width in terms of the partial widths of the normalized eigenchannels $\hat{\Gamma}_{c}=\left|\left\langle\hat{\phi}_{c} \mid \psi\right\rangle\right|^{2}$, and using the property that the cross-channel correlator is smaller by $1 / N$ than the autochannel correlator, we obtain in the limit of large $N$ [14] 


$$
c_{G}\left(x-x^{\prime}\right)=\overline{\overline{\hat{\Gamma}}_{1}(x) \tilde{\hat{\Gamma}}_{1}\left(x^{\prime}\right)},
$$

where $\hat{\Gamma}_{1}$ is the partial width of an arbitrary normalized channel. Remarkably, the peak correlator for a symmetric dot is not only universal but is also independent of the details of the channels in the external leads, including their number, the rate of tunneling into each and the correlations among them. We computed this function for both the orthogonal and the unitary cases from simulations of the simple GP defined by [15] $H(x)=H_{1} \cos x+H_{2} \sin x$ where $H_{1,2}$ are independent GOE (GUE) matrices. The results are presented in Fig. [1. We found that $c_{G}$ is fitted very well by a Lorentzian in the orthogonal case $(\beta=1)$ and by a squared Lorentzian in the unitary case $(\beta=2)$ :

$$
c_{G}\left(x-x^{\prime}\right)=\left[\frac{1}{1+\left(\bar{x}-\bar{x}^{\prime}\right)^{2} / \alpha_{\beta}^{2}}\right]^{\beta},
$$

where $\alpha_{1}=0.48 \pm 0.04$ and $\alpha_{2}=0.64 \pm 0.04$ with a $\chi^{2}$ per degree of freedom of $\approx 10^{-2}$.

We tested the GP prediction by studying the peak correlator in a disordered dot modelled by a two-dimensional Anderson Hamiltonian. We used a $27 \times 27$ lattice with a cylindrical geometry and introduced a parametric dependence by adding a step potential of strength $x$ along the symmetry axis. The left and right leads are represented by arrays $A^{l(r)}$ of $m_{1} \times m_{2}$ lattice points with total widths given by $\Gamma^{l(r)}=\sum_{\mathbf{r}_{i} \in A^{l(r)}} v_{i}^{2}\left|\Psi\left(\mathbf{r}_{i}\right)\right|^{2}$. In Fig. 目 we show the peak correlator for $1 \times 1$ and $4 \times 4$ leads, corresponding to leads with a single channel and with many correlated channels, respectively. We took $v_{i}=1$ but verified that different choices do not change the results, which are in excellent agreement with the GOP prediction. In the inset we show $\Gamma(\bar{x}) / \bar{\Gamma}$ for a typical member of the GOP and for one realization of the site energies in the Anderson model, using a single eigenfunction without any statistical averaging. As expected, this quantity exhibits irregular oscillations with a period comparable to the decorrelation distance along $\bar{x}$.

In order to break time-reversal symmetry, we applied a constant magnetic field along the symmetry axis, tuned such that the cylinder encloses a flux of $1 / 4$ flux unit. The parametric dependence was introduced either by a step potential as before or by closing the cylinder 
into a torus and applying a varying magnetic field perpendicular to the constant one. The peak correlators in both cases are also plotted in Fig. 1, and agree very well with the GUP prediction.

Although in principle all parametric correlators become universal upon the scaling (何), in practice this is not always experimentally feasible. The energy levels probed in a quantum dot are usually not the excited states for a fixed number of electrons, but the ground states for different numbers of electrons. Since the spacing between the conductance peaks is dominated by the charging energy $e^{2} / C$ which is much larger than the mean spacing $\Delta$, the scaling factor in (4) is difficult to measure. It is therefore important to derive a scaling that can be extracted directly from the conductance peak heights. For that purpose we define $\gamma_{\lambda}(x) \equiv\left\langle\hat{\phi} \mid \psi_{\lambda}(x)\right\rangle$ and consider its derivative

$$
\frac{\partial \gamma_{\lambda}}{\partial x}=\lim _{x^{\prime} \rightarrow x} \frac{1}{x^{\prime}-x} \sum_{\mu \neq \lambda} \frac{\left\langle\psi_{\mu}\left|H\left(x^{\prime}\right)\right| \psi_{\lambda}\right\rangle}{E_{\lambda}-E_{\mu}} \gamma_{\mu},
$$

given by first-order perturbation theory and where unprimed quantities are evaluated at $x$. The RMS of $\partial \gamma_{\lambda} / \partial x$ is then calculated in two steps. First, we perform the average over $H\left(x^{\prime}\right)$ at fixed $H(x)$, employing the conditional probability distribution [8] for which $\overline{H_{\mu \lambda}\left(x^{\prime}\right) H_{\nu \lambda}^{*}\left(x^{\prime}\right)}=\delta_{\mu \nu} a^{2}\left(1-f^{2}\right) / \beta$. Next we average over $H(x)$, taking advantage of the factorization of $P[H(x)]$ into a product of the eigenvalue and eigenfunction probability distributions. We obtain the following expression for the non-universal quantity $\kappa$ in the expansion of $f$ preceding (田):

$$
\kappa=\frac{1}{\beta Z N} \overline{\left|\frac{\partial \gamma_{\lambda}}{\partial x}\right|^{2}} / \overline{\left|\gamma_{\lambda}\right|^{2}}
$$

where the constant $Z \equiv\left(a^{2} / \beta N\right) \overline{\sum_{\mu \neq \lambda}\left(E_{\lambda}-E_{\mu}\right)^{-2}}=\left(4 / \beta \pi^{2}\right) \int_{0}^{\infty} d \epsilon\left[1-Y_{2}(\epsilon)\right] / \epsilon^{2}$, and $Y_{2}(\epsilon)$ is the two-point cluster function. Eq. (9) suggests a new scaling:

$$
x \rightarrow \bar{x}_{r}=\left(\frac{1}{\beta} \overline{\left|\frac{\partial \gamma_{\lambda}}{\partial x}\right|^{2}} / \overline{\left|\gamma_{\lambda}\right|^{2}}\right)^{1 / 2} x \equiv \sqrt{R} x .
$$

With this scaling, $f \approx 1-\left(\bar{x}_{r}-\bar{x}_{r}^{\prime}\right)^{2} / Z N$, and all correlators become universal functions of $\bar{x}_{r}-\bar{x}_{r}^{\prime}$. The scaling factor in (10) can be interpreted as the RMS of the eigenfunction 
rotation rate in analogy with the energy level velocity in the scaling (4). The quantity $Z$ above diverges for the GOP but is finite for the GUP $Z=2 / 3$, hence the scaling (10) is well-defined only in the unitary case. In this case (GUP) the ratio between the rotation rate and level velocity scaling factors is a universal constant, obtained from comparing (9) with the expression for $\kappa$ preceding (4)

$$
R / D=\pi^{2} / 3 \quad(\mathrm{GUP})
$$

The divergence of the scaling factor in (10) for the GOP notwithstanding, it is still possible to use this scaling if the derivative $\partial \gamma_{\lambda} / \partial x$ is replaced by $\Delta \gamma_{\lambda} / \Delta x$. This regularization translates into a small-spacing cutoff $\delta$ in $Z$ such that $\left|E_{\lambda}-E_{\mu}\right| / \Delta<\delta$ are excluded from the sum, resulting in a logarithmic divergence $Z \propto \log \delta$. For a small but finite $\Delta x$ we can then deduce the ratio between the two scaling factors for the GOP [14]:

$$
R / D=-\frac{\pi^{2}}{6} \log (\Delta \bar{x})+\text { Const. } \quad(\mathrm{GOP})
$$

Relation (12) is a universal function of $\Delta \bar{x}$, as we demonstrate in the inset of Fig. 1 and as is also confirmed in simulations of the Anderson model.

We now come back to the conductance peak correlator (6) and extract its short-distance behavior. We consider the quantity $\left(\Delta \Gamma_{\lambda}\right)^{2} \equiv\left[\Gamma_{\lambda}\left(x^{\prime}\right)-\Gamma_{\lambda}(x)\right]^{2}$ and calculate its average in first-order perturbation theory by expanding $\psi_{\lambda}\left(x^{\prime}\right)$ in $\psi_{\mu}(x)$. Averaging first over $H\left(x^{\prime}\right)$ we obtain

$$
\overline{\left(\Delta \Gamma_{\lambda}\right)^{2}} \approx \frac{2 a^{2}}{\beta}\left(1-f^{2}\right) \sum_{\mu \neq \lambda} \overline{\left[E_{\lambda}-E_{\mu}\right]^{-2}} \overline{\Gamma_{\lambda} \Gamma_{\mu}},
$$

where the remaining average is over $H(x)$. Using the GE relation $\overline{\Gamma_{\lambda} \Gamma_{\mu}}=$ $[\beta N / 2(N-1)]\left(\overline{\Gamma_{\lambda}^{2}}-{\overline{\Gamma_{\lambda}}}^{2}\right)$ and the scaling $(10)$ we find

$$
c_{G}\left(x-x^{\prime}\right) \approx 1-b_{\beta} \frac{R}{D}\left|\bar{x}-\bar{x}^{\prime}\right|^{2}
$$

where $b_{\beta}=\beta$ and $R / D$ is given by (11) for the GUP and by (12) for the GOP. When compared with the leading-order behavior of the squared Lorentzian (7) for the GUP case 
we find $\alpha_{2}=\sqrt{3} / \pi \approx 0.55$. The discrepancy with the value quoted below (7) indicates that (7), while being a good approximation, is not exact; indeed, higher order terms in (13) introduce odd powers of $\bar{x}-\bar{x}^{\prime}$. For the GOP, the non-analytic behavior of (14) at the origin indicates that (7) is not exact also in that case.

Using the perturbative expression (13), it is possible to express the rotation rate scaling factor $\sqrt{R}$ directly as the RMS of the normalized conductance peak velocity

$$
R=r_{\beta} \frac{1}{\bar{G}_{\lambda}^{2}} \overline{\left(\frac{\partial G_{\lambda}}{\partial x}\right)^{2}},
$$

where $r_{\beta}=1 / 4$ and for the GOP the derivative is discrete. One can calculate $R$ from the conductance peak data according to (15) and then use (11) or (12) to determine $D$ and thus the scaled parameter $\bar{x}$ that leads to universal correlations. A semiclassical calculation of $R$ 14 for a ballistic electron in a magnetic field $(x=B)$ leads to an estimate of the correlation field $B_{c}=R^{-1 / 2} \propto\left(2 m E \mathcal{A} / \hbar^{2}\right)^{1 / 4}\left(\Phi_{0} / \mathcal{A}\right)$, where $\mathcal{A}$ is the area of the dot and $\Phi_{0}$ is the flux unit.

Finally, we computed the peak correlator for an asymmetric dot using (11), this time with $\Gamma^{l} \neq \Gamma^{r}$. The left and right leads each have their own channel correlation matrix with no correlation between them for a sufficiently large separation. Fig. \& displays the GP universal predictions for $c_{G}$ for single-channel symmetric leads $\left(\overline{\Gamma^{l}}=\overline{\Gamma^{r}}\right)$, and a comparison with Anderson model simulations. Eq. (7) still provides a good fit but with $\alpha_{1}=0.37 \pm 0.04$ and $\alpha_{2}=0.54 \pm 0.04$. We also find that Eq. (14) holds but with $b_{1}=7 / 4$ and $b_{2}=3$ [14]. The rotation scaling factor is calculated from (15) where now $r_{1}=1 / 7$ and $r_{2}=5 / 24$ [14. For asymmetric single-channel leads $\left(\overline{\Gamma^{l}} \neq \overline{\Gamma^{r}}\right), c_{G}$ is found to be intermediate between the symmetric single-channel leads correlator and the symmetric dot correlator. The latter is experimentally measurable in an asymmetric dot whose leads are made very asymmetric, since the conductance peak is then approximately proportional to the dominating width. For symmetric leads with many equivalent channels the peak correlator also approaches the width correlator [14].

In conclusion, we computed the universal conductance peak correlator and obtained a 
good approximation (7) of its functional form. We derived the rotation rate scaling which is useful when the level velocity scaling factor is not measurable and which can be directly calculated from the conductance peaks data using (15). Since the submission of this Letter, our prediction (17) has been confirmed experimentally for broken time-reversal symmetry [16]. We also remark that the width correlator (6,7) is identical to the correlator of wavefunction intensity at a fixed spatial point which can be measured in microwave cavity experiments 17 as a function of shape. This work was supported in part by the Department of Energy Grant DE-FG02-91ER40608. We acknowledge C.M. Marcus and A.D. Stone for useful discussions. 


\section{REFERENCES}

[1] M.A. Kastner, Rev. Mod. Phys. 64, 849 (1992).

[2] P.L. McEuen et al. , Phys. Rev. Lett. 66, 1929 (1991).

[3] R.A. Jalabert, A.D. Stone, and Y. Alhassid, Phys. Rev. Lett. 68, 3468 (1992).

[4] V.N. Prigodin, K.B. Efetov and S. Iida, Phys. Rev. Lett. 71, 1230 (1993); E.R. Mucciolo, V.N. Prigodin, and B.L. Altshuler, Phys. Rev. B 51, 1714(1995).

[5] R.A. Jalabert, H.U. Baranger and A.D. Stone, Phys. Rev. Lett. 65, 2442 (1990); H.U. Baranger, R.A. Jalabert and A.D. Stone, Chaos 3, 665 (1993).

[6] K.B. Efetov, Phys. Rev. Lett. 74, 2299 (1995).

[7] C.M. Marcus, R.M. Westervelt, P.F. Hopkins and A.C. Gossard, Phys. Rev B 48, 2460 (1993); I.H. Chan, R.M. Clarke, C.M. Marcus, K. Campman and A.C. Gossard, Phys. Rev. Lett. 74, 3876 (1995).

[8] Y. Alhassid and H. Attias, Phys. Rev. Lett. 74, 4635 (1995).

[9] B.D. Simons and B.L. Altshuler, Phys. Rev. Lett. 70, 4063 (1993); Phys. Rev. B 48, 5422 (1993).

[10] Y. Meir, N. Wingreen and P.A. Lee, Phys. Rev. Lett. 66, 3048 (1991).

[11] C.W.J. Beenakker, Phys. Rev. B 44, 1646 (1991).

[12] A.M. Lane and R.G. Thomas, Rev. Mod. Phys. 30, 257 (1958).

[13] M. Wilkinson, J. Phys. A 22, 2795 (1989).

[14] Y. Alhassid and H. Attias, to be published.

[15] E.J. Austin and M.Wilkinson, Nonlinearity 5, 1137 (1992).

[16] J.A. Folk, S.R. Patel, S.F. Godijn, A.G. Huibers, S.M. Cronenwett, C.M. Marcus, K. 
Campman and A.C. Gossard, preprint (September 1995).

[17] S. Sridhar, D. Hogenboom and B. Willemsen, J. Stat. Phys. 68, 239 (1992). 


\section{FIGURES}

FIG. 1. Universal form of the width correlator (6) as a function of the scaled parameter (4). Top: GP calculations (diamonds) and their best fit to (7) (dashed). For comparison we also plot the GUP result on the left and the GOP result on the right (dotted). Insets: the universal ratio $R / D$ computed with a finite $\Delta \bar{x}$ using simulations of the GP (diamonds with statistical errors) and the Anderson model (squares and circles). The dashed line in the left inset is the theoretical prediction (12). Bottom: Anderson model simulations. Left: leads of $1 \times 1$ (pluses) and $4 \times 4$ (squares) points with a varying step potential. Right: a magnetic flux of $\frac{1}{4} \Phi_{0}$ is applied for $1 \times 1$ leads with an additional varying magnetic field (crosses) and $4 \times 4$ leads with a varying step potential (circles). Insets: typical width fluctuations for a single eigenfunction before the statistical averaging for the GP (solid) and Anderson model (dashed).

FIG. 2. Universal form of the peak correlator in asymmetric dots with symmetric single-channel leads for both orthogonal and unitary symmetries. The GP predictions (dashed) are compared with Anderson model simulations (pluses and crosses as in Fig. 1). Shown by dotted lines is the width correlator which also describes the conductance correlator for highly asymmetric leads. 
Orthogonal

Unitary

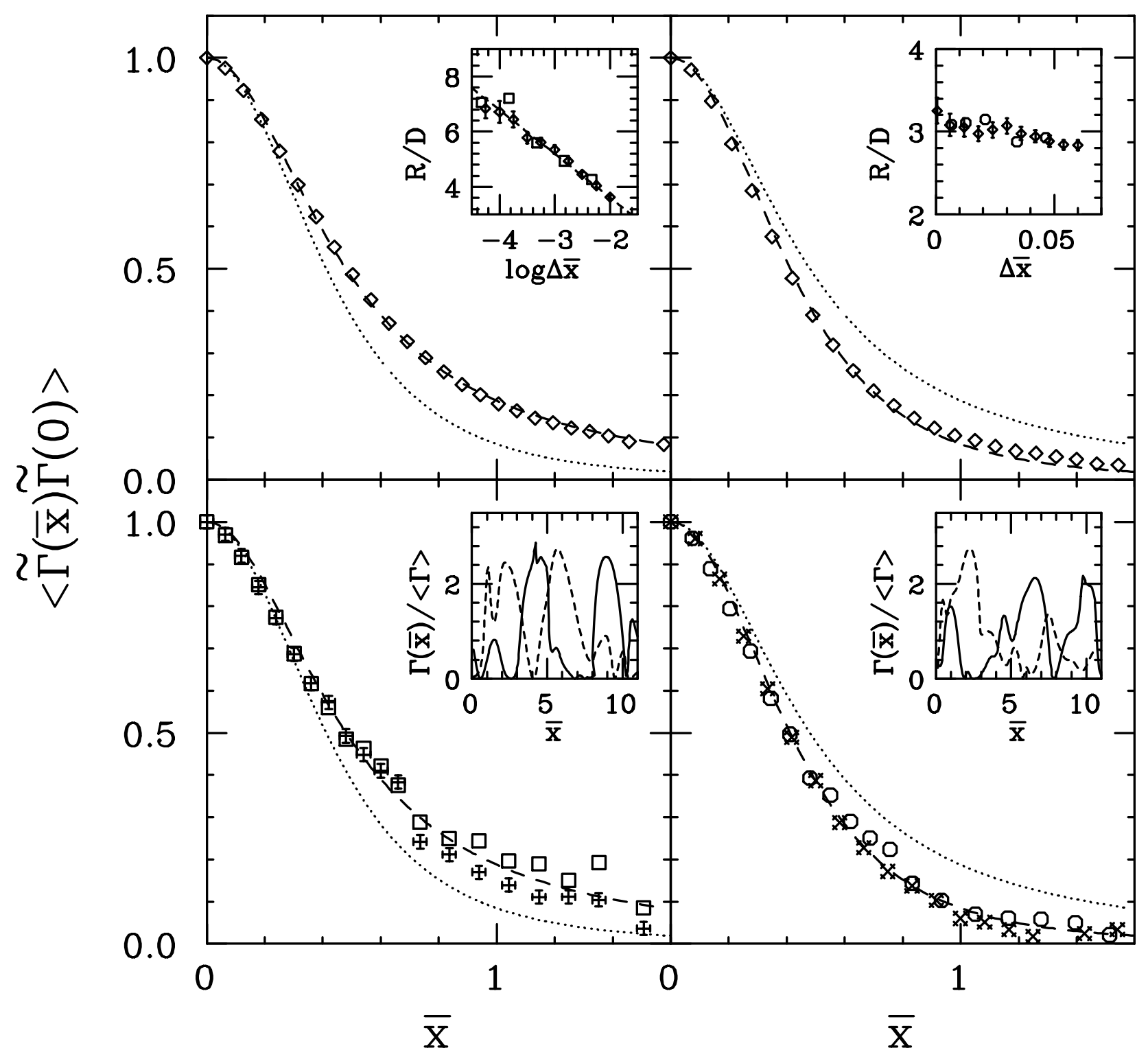




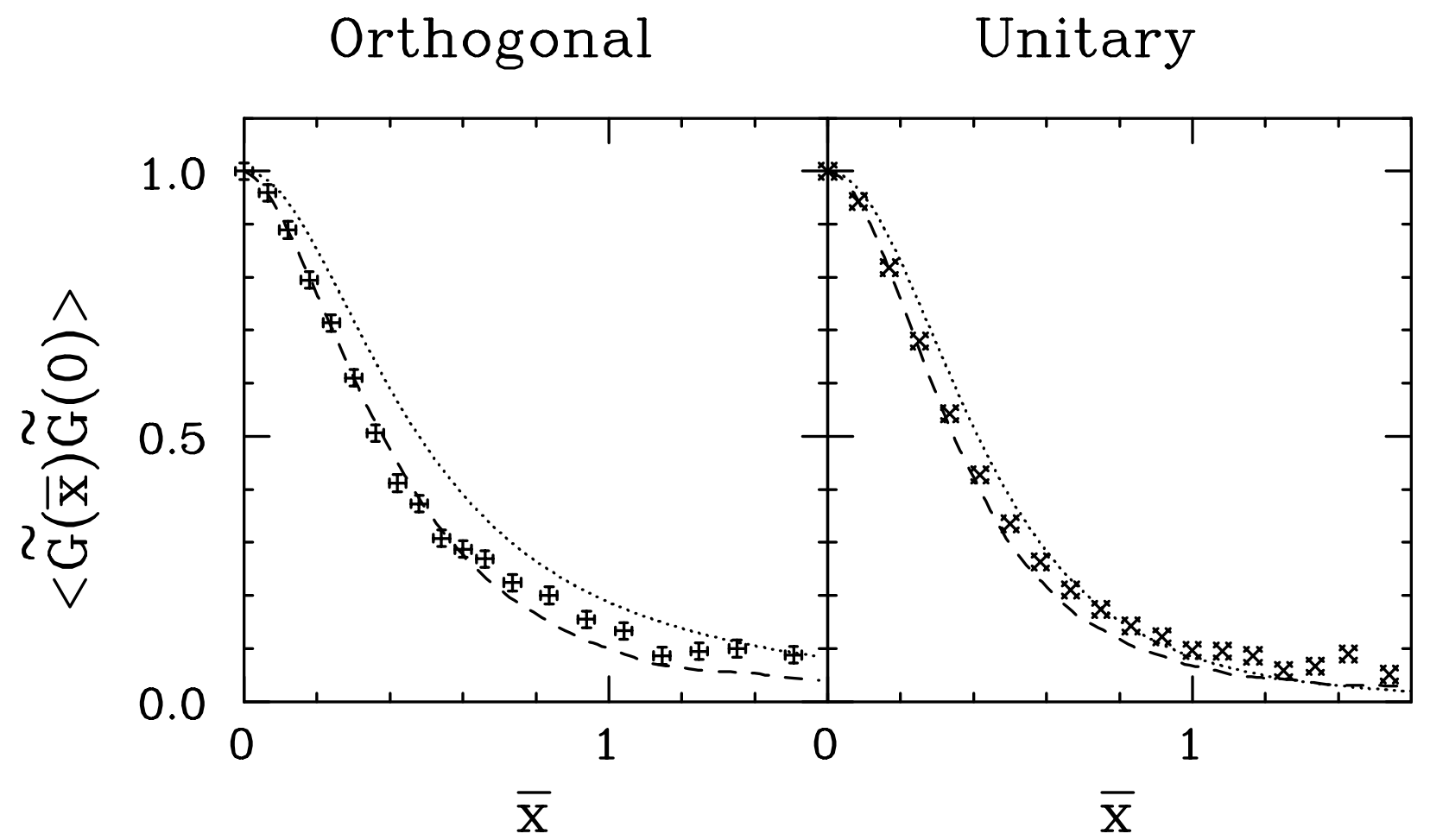

